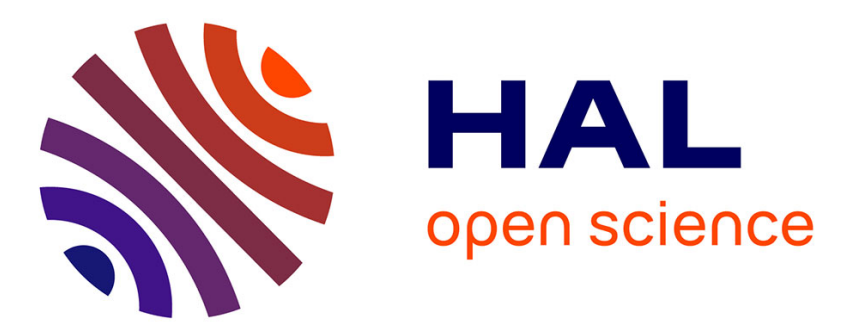

\title{
Tolerance Analysis of a Deformable Component Using the Probabilistic Approach and Kriging-Based Surrogate Models
}

Pierre Beaurepaire, Cécile Mattrand, Nicolas Gayton, Jean-Yves Dantan

\section{- To cite this version:}

Pierre Beaurepaire, Cécile Mattrand, Nicolas Gayton, Jean-Yves Dantan. Tolerance Analysis of a Deformable Component Using the Probabilistic Approach and Kriging-Based Surrogate Models. ASCEASME Journal of Risk and Uncertainty in Engineering Systems, Part A: Civil Engineering, 2018, 4 (3), pp.04018028. 10.1061/AJRUA6.0000979 . hal-02367670

\author{
HAL Id: hal-02367670 \\ https://hal.science/hal-02367670
}

Submitted on 18 Nov 2019

HAL is a multi-disciplinary open access archive for the deposit and dissemination of scientific research documents, whether they are published or not. The documents may come from teaching and research institutions in France or abroad, or from public or private research centers.
L'archive ouverte pluridisciplinaire HAL, est destinée au dépôt et à la diffusion de documents scientifiques de niveau recherche, publiés ou non, émanant des établissements d'enseignement et de recherche français ou étrangers, des laboratoires publics ou privés. 


\title{
Tolerance analysis of a deformable component using the probabilistic approach and Kriging-based surrogate models
}

\author{
P. Beaurepaire,${ }^{1}$ C. Mattrand, ${ }^{2}$ N. Gayton, ${ }^{3}$ J.-Y. Dantan ${ }^{4}$
}

\section{ABSTRACT}

Tolerance analysis is a key issue in proving the compatibility of manufacturing uncertainties with the quality level of mechanical systems. For rigid and isostatic systems, multiple methods (worst case, statistical or probabilistic approaches) are applicable and well established. Recent scientific developments have brought enhancements for rigid over-constrained systems, using probabilistic and optimization based methods. The consideration of non-rigid systems is more complex, since large-scale numerical model must be taken into account for an accurate prediction of the quality. The aim of the present paper is the illustration of the probabilistic tolerance analysis approach for an industrial application

\footnotetext{
${ }^{1}$ Assistant professor, Université Clermont Auvergne, CNRS, SIGMA-Clermont, Institut Pascal, Campus de Clermont-Ferrand / Les Cézeaux, 27 rue Roche Genès, CS 20265, 63175 Aubière Cedex, France, pierre.beaurepaire@sigma-clermont.fr

${ }^{2}$ Assistant professor, Université Clermont Auvergne, CNRS, SIGMA-Clermont, Institut Pascal, Campus de Clermont-Ferrand / Les Cézeaux, 27 rue Roche Genès, CS 20265, 63175 Aubière Cedex, France, cecile.mattrand@sigma-clermont.fr

${ }^{3}$ Professor, Université Clermont Auvergne, CNRS, SIGMA-Clermont, Institut Pascal, Campus de Clermont-Ferrand / Les Cézeaux, 27 rue Roche Genès, CS 20265, 63175 Aubière Cedex, France, nicolas.gayton@sigma-clermont.fr

${ }^{4}$ Professor, LCFC, Arts et Métiers, ParisTech Metz, 4 rue Augustin Fresnel, 57078 Metz Cedex 3, France, jean-yves.dantan@ensam.eu
} 
involving deformable parts. The distributions associated with the dimensions of

the components are identified using real components collected from the assembly lines. A nonlinear finite element model is used to predict the mechanical behavior. A reliability analysis is performed in order to compute the defect probability and estimate the quality of the products. A Kriging-based surrogate model is used to reduce the numerical efforts required for the reliability analysis.

Keywords: Tolerance analysis; Defect probability estimation; System reliability; Kriging-based surrogate model, Wiping system

\section{INTRODUCTION}

Engineers are aware that uncertainties in the dimensions of manufactured products cannot be avoided, i.e. mechanical components manufactured on the same assembly line using the same tools and the same raw materials have slightly different shapes; and their dimensions are also different from the designer's target. Tolerance analysis offers a rational framework to study such uncertainties, and enables engineers to guarantee that the quality resulting from the production process remains acceptable. Consequently, production wastage and global manufacturing costs are considerably reduced.

It is assumed that the behavior of a mechanical system is fully characterized by a finite set of parameters $\boldsymbol{X}$, which are associated with the deviations between the ideal geometry and the geometry of real components; the shape of the components is parameterized; and the vector $\boldsymbol{X}$ has a finite size. The response of the system $Y$ is described using the functional characteristics (Nigam and Turner 1995); its expression is of the form:

$$
Y=f(\boldsymbol{X})
$$

where $f$ denotes the response function of the mechanical component. 
Tolerance analysis can be performed by considering the upper and lower bounds of the functional characteristic expressed in Equation (1). The system is functional as long as its response is between these two bounds. Two strategies are applicable to deal with the geometric deviations (Chase and Parkinson 1991; Greenwood and Chase 1987; Nigam and Turner 1995).

1. With the worst case approach, each dimension $X_{i}$ is characterized by an upper and a lower bound; and the configuration leading to the worse performance is identified. The tolerance intervals of the dimensions are adjusted in order to guarantee that the component is functional for the worst case (i.e. that the functional characteristics are between the predefined bounds for all the possible values of $\boldsymbol{X}$ ).

2. The statistical approach consists of introducing a probabilistic model for the dimensions, and uncertainties are subsequently propagated to the response of the mechanical component. The function characteristic may be outside the predefined bounds; this is tolerated as long as such events remain rare and the frequency of occurrence is controlled. The objective of the tolerance analysis is the determination of this occurrence probability, which is referred to as the defect probability. It provides a metric associated with the quality of the production, which is often expressed in parts per million (ppm) or in parts per billion (ppb) for systems manufactured by Valeo VWS (the industrial partner in this study). The worst case approach is more conservative, which leads to excessively tight tolerance intervals and higher manufacturing costs (Hong and Chang 2002; Roy et al. 1991); the statistical approach is therefore used here.

During the last two decades, three main issues have been addressed by the tolerance analysis community for the statistical approach. Issue 1 concerns the 
modeling of random dimensions by probability distributions. Tolerance analysis is commonly performed in the design stage to predict defect probability, and the tolerance intervals are adjusted to meet predefined quality requirements. One of the major challenges is the lack of information at this stage, since parts are not available and it is hence not possible to use measurements of the uncertain dimensions to identify their distributions. As a result, assumptions must be introduced into the uncertainty model (using, for instance, feedback obtained with similar components). A possible strategy consists of modeling each dimension with a uniform distribution inside the tolerance interval (Greenwood and Chase 1987). However, this approach may be conservative, and alternative strategies are applicable, such as the use of centered or shifted Gaussian distributions (Evans 1975; Scholtz 1995). The uncertainty model may also be defined by means of a dynamic approach (Gayton et al. 2011) when considering batch production. A multi-level model is introduced and the dimensions are modeled using Gaussian distributions. The parts in the same batch have identical mean and standard deviation for all their dimensions. These moments are modeled as random variables, which introduces a second level of uncertainty (Gayton et al. 2011; Scholtz 1995). All these models require assumptions which have considerable consequences on defect probability prediction. The second major issue (issue 2) is the tolerance analysis in case of over-constrained mechanical systems. Equation (1) is not applicable to such problems, as the functional requirement involves the uncertain dimensions of the components, but also gap variables, which may be associated with the distance between the components of the assembly. It is not possible for the designer to set the value of these variables, and they are not characterized by a probability density function. As the gaps cannot be modeled using random variables nor design variables, they are referred to as free variables in this manuscript. The formulation of the tolerance analysis problem with gap variables is described e.g. in 
(Dantan and Qureshi 2009). This is a challenging task, as the identification of an explicit expression of the functional characteristic is in general not possible. For over-constrained systems, multiple contact configurations are possible, leading to multiple candidate values of the functional characteristic. Specific methods have been proposed to identify appropriate contact configurations and compute the defect probability (Dumas et al. 2015; Qureshi et al. 2012). However, tolerance analysis of over-constrained systems remain a challenging task. The last issue (issue 3) concerns the tolerance analysis of systems with deformable parts; the compliance of the components is explicitly considered by introducing a mechanical model, obtained for instance using the finite element method (see e.g. (Gordis and Flannelly 1994; Liu and Hu 1996; Söderberg et al. 2006)). Liu and Hu (1997) showed that dimensional variation has little effect on the stiffness of the components, and a deterministic model can be used for their mechanical behavior. This strategy, known as the influence coefficients method, has been applied with success to multiple problems, see e.g. (Dahlström and Lindkvist 2006; Li et al. 2004; Lindau et al. 2015). The method is applicable only if (i) the coefficient of variation associated with the uncertain dimensions is sufficiently small (in order they have no effects on the stiffness matrix); (ii) the materials behavior is linear (or the strain is sufficiently small to have a linear material behavior).

The Monte Carlo Simulation is widely used to compute the probability of defect, as this method is applicable to non-linear models and non-Gaussian distributions. However, the Monte Carlo method requires considerable numerical efforts when the defect probability is low or when a large scale model is used. Advanced reliability methods, such as the First and Second Order Reliability Method (FORM, SORM) or importance sampling can be used to reduce these numerical efforts (Lemaire 2010).

The present paper deals with the implementation of an industrial tolerance 
analysis application. The problem is the prediction of the defect probability of a deformable wiper blade system subjected to shape and material uncertainty. In this work, the three issues discussed above are considered with great attention. The manufacturing of these components started a few years ago and it is therefore possible to directly measure their dimensions. The problem of the identification of the distribution (issue 1) is hence simplified, as data are available and can be used to identify the most suitable distribution for each dimension. The additional complexity introduced by free variables (issue 2) is addressed by calibrating a response surface, which is subsequently used to eliminate these variables with reduced computational efforts. For the tolerance analysis of such a component, the functional requirements are obtained directly from the structural response. The influence of the uncertain dimensions on the stiffness matrix cannot be neglected (and has to be fully considered). The simplifying hypothesis used in the literature for the tolerance analysis of deformable components (issue 3) is not applicable here, and multiple finite element simulations are required to perform the reliability analysis and compute the defect probability. An advanced simulation method is used to perform this analysis with acceptable numerical efforts; it relies on the use of Kriging-based surrogate model (Echard et al. 2011; Echard et al. 2013; Fauriat and Gayton 2014).

This manuscript is structured as follows: the considered industrial problem in described in Section 2; the stochastic structural model is described in Section 3 with a presentation of the modeling of uncertainties from profile measurements. The proposed surrogate model-based methodology is next discussed in the fourth section before presenting the results in Section 5. The article closes with conclusions and perspectives in Section 6.

\section{DESCRIPTION OF THE INDUSTRIAL PROBLEM}


This study concerns the tolerance analysis of a wiper system. Such components are used in the automotive industry to remove water and debris from the windshield. The methods developed here are applied to flat blade technology. The blade is fixed at its center to the wiper arm, which applies an alternating rotation movement (see Figure 1) and maintains the contact between the blade and the windshield.

The blade is mainly composed of metallic splines and of a rubber profile that is the focus of this paper. The shape of the splines matches the curvature of the windshield; they provide sufficient stiffness to the assembly, preventing an uneven distribution of the pressure at the contact between the blade and the windshield. The rubber profile includes multiple sub-components (see Figure 2):

- the lips ensure wiping and windshield cleaning;

- the hinge, which controls the deformation of the blade and the contributes to reverse the blade (when the wiper reaches the end of its travel and turns back);

- the heel, which locks the fir to the blade assembly.

During wiping, the profile is considerably strained; the fir and the heel come into contact as shown in Figure 2. The mechanical deformation of the rubber profile during the wiping cycle depends on the tip force, the friction coefficient between the windshield and the rubber, the material properties and the geometrical characteristics. A good control of blade profile deformation prevents:

- fast deterioration of the rubber leading to ridge defect on the windshield as shown in Figure 3a;

- return defects generated by particular geometrical conditions of the rubber profile as shown in Figure 3b. 
The performance of the system is evaluated from the structural response, i.e. fluid-structure interactions are not considered here, and therefore the multiphysics problem is transformed into a mechanical problem. The two first performance criteria of the wiper system are determined using the contact angle $\alpha$ (i.e. the angle between the lips of the fir and the windshield) and the locking angle $\beta$ (measured at the contact point between the fir and the heel, as shown in Figure 2). The maximum strain $\epsilon_{\max }$ of the rubber hinge is used as a third performance criterion, since it may be an indicator of the aging of the rubber.

This paper is focused on the investigation of the consequences of geometrical and material uncertainties on the performance of a wiper system. Only the uncertainties associated with the rubber profile are considered. Consequently, the aim of this paper is the evaluation of the system probability that $\alpha, \beta$ and $\epsilon_{\max }$ fall outside functional ranges, each quantity depending on random geometry, characterized by parameters grouped in vector $\boldsymbol{D}$, and the material properties, characterized by parameters grouped in vector $\boldsymbol{P}$.

This work also enables us to obtain feedback on the actual distribution of the dimensions of the wiper blade. This is an important matter, since in practice designers lack this information to estimate the quality level associated with a component. The study provides an opportunity to analyze real part data, identify the distribution associated with the various dimensions, determine whether the assumptions usually made during design are realistic, etc. The dependence between the dimensions is an interesting element, too. Indeed, the profiles are manufactured using an extrusion process, which introduces correlation between the dimensions. The information collected here may be re-used in the future in the definition of the random variable set for wiper profiles manufactured using the same process. 


\section{STOCHASTIC STRUCTURAL MODEL}

\section{Probabilistic model}

\section{Characterization of uncertain parameters}

The components are manufactured using a rubber extrusion process, and the profiles are subsequently cut to obtain blades of the required length. The uncertainties associated with the length of the blade are not considered here, and thus the geometry of the component is defined by the cross-section of the blade. The reference cross-section, as it appears for instance in the computer-aided design model, is shown in Figure 4a. The manufacturing process introduces unavoidable geometrical deviations, and the actual geometry of a wiper blade differs from the reference geometry. A manufactured part is shown in Figure 4b and geometrical deviations are perceptible. The shape of the rubber profile is frequently controlled in the factories to quantity these geometric deviations; a video measurement tool is used to avoid the part deformation during its size control. The shape of the observed cross-section is complex, and the preparation of a geometrical model capturing fully the deviation with respect to the drawings would be a challenging task. A simplified non-ideal cross-section is introduced; it is fully described using a finite number of parameters $\boldsymbol{D}$ as shown in Figure 5. In total, 44 different quantities are determined to characterize the reference cross-section of the blade (length and width at various locations, fillet radii, etc). A probabilistic approach is used and a random variable is introduced for each geometrical parameter.

It is assumed that the uncertainties can be fully characterized using the linear correlation matrix and marginal distributions. Alternative strategies may be considered to account for the correlation between the dimension, such as for instance copulas (see e.g. (Mai and Scherer 2012; Schölzel and Friederichs 2008)). However, such approaches are not used here. 
Initially, a set of candidate distributions is arbitrarily selected. Only four candidate distributions are use here but additional distributions may be included without modifying the proposed procedure. In the study, the dimensions may follow either a uniform, exponential, normal or lognormal distribution. In each case, it is necessary to identify the parameters of the distribution leading to the best match with the data obtained from the factory. These parameters are obtained by maximizing the likelihood function, which is expressed as:

$$
L\left(D_{i}^{(1)}, D_{i}^{(2)}, \ldots, D_{i}^{(45)}, \boldsymbol{p}, \mathcal{D}\right)=\prod_{j=1}^{45} f_{D_{i}}\left(D_{i}^{(j)}, \boldsymbol{p}, \mathcal{D}\right)
$$

where $D_{i}^{(j)}, j=1 \ldots 45$ denotes the measurements available for the $i^{\text {th }}$ dimension of the wiper blade, which are used to identify the distribution of the corresponding random variable; in total, 45 dimension measurements are used to identify the distributions. $\boldsymbol{p}$ is a vector grouping all the distribution parameters (e.g. mean, standard deviation, bounds), $f_{D_{i}}$ denotes the probability density function of the random variable $D_{i}$ and $\mathcal{D}$ represents the considered distribution (i.e. either the normal, uniform, exponential or lognormal distribution). The value of the terms of $\boldsymbol{p}$ is selected such that $L$ is maximized.

The most suitable distribution is then selected using the Akaike Information Criterion (AIC) (Akaike 1974):

$$
A I C\left(D_{i}^{(1)}, \ldots, D_{i}^{(45)}, \hat{\boldsymbol{p}}, \mathcal{D}\right)=-2 \ln L\left(D_{i}^{(1)}, \ldots, D_{i}^{(45)}, \hat{\boldsymbol{p}}, \mathcal{D}\right)+2 q(\mathcal{D})
$$

where $\hat{\boldsymbol{p}}$ denotes the optimal value of the distribution parameters (which maximize Equation (2)), and $q$ is the number of parameters associated with the distribution. For an exponential distribution, $q$ is equal to one (and in this case $\hat{\boldsymbol{p}}$ is a scalar); otherwise $q$ is equal to two (and $\hat{\boldsymbol{p}}$ is a vector with two terms). The distribution 
$\mathcal{D}$ associated with the maximum value of the $A I C$ leads to the best fit with the available data, and is subsequently used in the probabilistic model.

This operation is repeated for all the dimensions considered and, in total, 44 distributions are identified using the procedure described above. It is observed that either the normal distribution or uniform distribution maximize the AIC for most of the dimensions; the lognormal distribution is occasionally used, but it remains rare; the exponential distribution is never used.

The manufacturing process has a strong influence on the dependence between the uncertain parameters, as physical phenomena are involved and impact all the dimensions. For instance, the rubber may expand after extrusion, causing a positive correlation between the dimensions $H_{2}$ and $H_{4}$ indicated in Figure 5, whereas it causes a negative correlation between the dimensions $H_{1}$ and $H_{2}$. Figure 6 represents a scatterplot of these dimensions, the correlation between the dimensions is clearly visible. In this work, samples of the uncertain parameters are available and for each measurement, all the dimensions are determined on the same part. Thus, the correlation matrix can be directly computed from the measurements.

The vector $\boldsymbol{P}$ gathers the uncertainties associated with the material parameters. These coefficients are used to define a probabilistic hyperelastic model of the mechanical behavior of rubber. In total, two independent random variables are used to characterize the material uncertainties. The details of this model are not discussed here for confidentiality reasons. 
Isoprobabilistic transformation

Most reliability algorithms are applied in the so-called standard normal space, where all the random variables are independent and have a standard normal distribution, with a zero mean and a unitary standard deviation. An isoprobabilistic transformation is applied to each random variable; it is expressed as:

$$
z_{i}=\Phi^{-1}\left(F_{D_{i}}\left(D_{i}\right)\right) \text { for } i=1, \ldots, 44
$$

where $\Phi^{-1}$ denotes the inverse of the standard normal cumulative density function, $F_{D_{i}}$ is the cumulative distribution function associated with the variable $D_{i}$ and $z_{i}$ denotes the random variables expressed using its original distribution and its counterpart in the standard normal space, respectively.

In case the variables $D_{i}$ and $D_{j}$ are correlated before the transformation described in Equation (4), the variables $z_{i}$ and $z_{j}$ are correlated as well, and $\rho_{i j}^{\prime}$ denotes their correlation coefficient. The approximation of $\rho_{i j}^{\prime}$ available in (Liu and Der Kiureghian 1986) are used here.

In the standard normal space the covariance matrix and the correlation matrix are identical and defined as:

$$
\Sigma^{\prime}=\left[\rho_{i j}^{\prime}\right]_{1 \leqslant i \leqslant 44,1 \leqslant j \leqslant 44}
$$

The Karhunen-Loève (Karhunen 1947; Loève 1977) transform is used to decorrelate the random variable, it is expressed as:

$$
\boldsymbol{z}=\sum_{i=1}^{44} \xi_{i} \sqrt{\lambda_{i}} \boldsymbol{\phi}_{i}
$$

where $\boldsymbol{z}=\left[z_{1}, \ldots, z_{44}\right] ; \xi_{i}, i=1 \ldots 44$ denotes independent variables with a standard normal distribution, and $\lambda_{i}$ and $\phi_{i}$ denote respectively the eigenvalues and the 
eigenvectors associated with the matrix $\Sigma^{\prime}$.

In Equation (6), the eigenvalues are sorted in descending order, and hence the first few terms have a major contribution to the variance of the set $\boldsymbol{z}$. Figure 7 shows the percentage of explained variance, expressed in terms of the total number of considered eigenvalues. The Karhunen-Loève expansion can be truncated to reduce the total number of random variables involved in the problem, with reduced loss of accuracy regarding the covariance matrix of the random variable set. In this work, the Karhunen-Loève expansion is performed using the 10 first terms of Equation (6). We have $\sum_{i=1}^{10} \lambda_{i} / \sum_{i=1}^{44} \lambda_{i}>0.95$ and hence at least $95 \%$ of the variance of $\boldsymbol{z}$ is accounted for. The Karhunen-Loève expansion allows us to considerably reduce the dimensionality of the problem because the random variables are strongly correlated.

\section{Mechanical model}

A finite element model is prepared to predict the behavior of the blade and determine the contact angle $\alpha$, the locking angle $\beta$ and the maximum strain $\varepsilon_{\max }$. The boundary conditions applied to the structure need to be identified to set up the mechanical model. The reaction forces at the contact and the coefficient of friction between the blade and the windshield are the key inputs.

The contact forces are not constant along the wiper blade; they are therefore expressed in terms of the $\mathrm{x}$-coordinate. This variation of the contact forces is caused by the geometry of the wiping blade and the curvature of the windshield. It is observed that the maximum force is obtained in the middle of the blade, as the connection with the wiper arm is situated in this location. Figure 8a shows the distribution of the forces with respect to the $\mathrm{x}$-coordinate. These curves are obtained via a beam model; the details of its implementation are not discussed herein. The inclination of the wiper on the windshield causes aerodynamic effects 
on its movement. During the upward movement, the wiping benefits from positive airflow effects, and hence the applied load is lower. However, the downward movement is adversely affected by the airflow, which causes a higher load. As a result, two distinct types of forces are presented. The first curve (dashed black line) corresponds to the efforts during wiping in the upwards direction and the second curve (continuous grey line) to those in the downward direction. The maximum load $F_{\max }$ is reached at the center of the blade for the wiping in the upwards direction; the minimum load $F_{\min }$ is reached at two different positions for the wiping in the upwards direction.

The friction coefficient between the blade and the windshield varies along the wiper length as well, and is influenced by the velocity of the blade. The friction coefficient increases as the velocity decreases. During wiping, the outer portion covers a greater distance and therefore has a higher speed than the inner part. In the mechanical model, the friction coefficient $\mu$ follows a linear curve along the length of the wiper, as shown in Figure 8b. Indeed, the difference in values is explained by the fact that the speed is not the same along the whole length of the wiper. In practice, stick-slips may be observed, leading to a more complex behavior. The linear evolution of the coefficient of friction is a firstorder approximation and more complex models are not used here for the sake of simplicity. A change in the friction model would not affect the tolerancing methodology. The minimum and maximum values of the coefficient of friction $\mu_{\min }$ and $\mu_{\max }$ are reached at the ends of the blade.

The finite element method is accurate only if the elements have roughly the same size in all directions (i.e. the same length, width and height). The total length of the wiper blade is approximately 100 times greater than its width or eight. A three dimensional mesh would hence include a large number of elements. The model is non-linear since the rubber has hyperelastic properties, large dis- 
placements are observed and the contact with the windshield is accounted for. Hence, the problem is not suitable for the application of a three-dimensional model, as it would involve multiple inversions of a large scale stiffness matrix. A simplified two-dimensional mechanical model is used instead, as shown in Figure 9. Each simulation is associated with a specific position on the blade, i.e. with a specific $\mathrm{x}$-coordinate, since the boundary conditions are expressed in terms of the x-coordinate. Thus, the corresponding load and coefficient of friction need to be injected into the model; they are selected as shown in the curves represented in Figure 8. Multiple simulations are performed in order to account for the variation in the response of the blade with respect to the $\mathrm{x}$-coordinate, and wiping in the upwards and the downwards directions also needs to be accounted for. Each finite element simulation takes one to ten minutes, depending on the non-linearity (contact configuration, material parameters, etc.).

\section{Performance functions}

Performance functions are introduced for the reliability analysis; their formulation involves the functional requirements. The value of the performance function is less than zero in the failure domain, i.e. in the case where one of the functional requirements is not fulfilled, and this function is greater than zero otherwise. As discussed in Section 3, the boundary conditions are expressed with respect to the $\mathrm{x}$-coordinate in the blade. As a result, the performance functions are also expressed in terms of the $\mathrm{x}$-coordinate. The wiper blade is assumed to be functional at a given position with $x$ as a coordinate if the maximum strain is below a predefined value; the contact angle and the locking angle are within a predefined range. Hence, five normalized performance functions are introduced, they are defined as: 


$$
\begin{aligned}
& g_{1}(\boldsymbol{D}, \boldsymbol{P}, x)=\frac{\varepsilon_{\text {max }}^{u}-\varepsilon_{\max }(\boldsymbol{D}, \boldsymbol{P}, x)}{\varepsilon_{\max }^{u}} \\
& g_{2}(\boldsymbol{D}, \boldsymbol{P}, x)=\frac{\alpha(\boldsymbol{D}, \boldsymbol{P}, x)-\alpha^{l}}{\alpha^{l}} \\
& g_{3}(\boldsymbol{D}, \boldsymbol{P}, x)=\frac{\alpha^{u}-\alpha(\boldsymbol{D}, \boldsymbol{P}, x)}{\alpha^{u}} \\
& g_{4}(\boldsymbol{D}, \boldsymbol{P}, x)=\frac{\beta(\boldsymbol{D}, \boldsymbol{P}, x)-\beta^{l}}{\beta^{l}} \\
& g_{5}(\boldsymbol{D}, \boldsymbol{P}, x)=\frac{\beta^{u}-\beta(\boldsymbol{D}, \boldsymbol{P}, x)}{\beta^{u}}
\end{aligned}
$$

where $\varepsilon_{\max }, \alpha$ and $\beta$ denote the functional requirements, i.e. the maximum strain, the contact angle and the locking angle, respectively; these functions are evaluated in terms of the position along the rubber profile $x$ and in terms of a particular value of the uncertain dimensions $\boldsymbol{D}$ and material parameters $\boldsymbol{P}$. $\varepsilon_{\max }^{u}$ is the maximum admissible strain; $\alpha^{u}$ and $\beta^{u}$ are the maximum admissible contact and locking angles, respectively; $\alpha^{l}$ and $\beta^{l}$ are the minimum admissible contact and locking angles, respectively.

The functions described in Equation (7) can be used to describe the behavior of the wiper blade locally, at the point with the coordinate $x$, whereas the functionality of the wiper must be determined globally at the level of the system. For a set of random variables $\boldsymbol{D}, \boldsymbol{P}$ (geometric and material, respectively), the profile is functional if it leaves no visible wiping defects on the windshield, i.e. all the performance functions must be greater than zero for all the possible values of $x$.

To solve the reliability problem, the performance functions can be formulated without dependence on the x-coordinate. They are expressed as:

$$
G_{i}(\boldsymbol{D}, \boldsymbol{P})=\min _{x_{\min }<x<x_{\max }} g_{i}(\boldsymbol{D}, \boldsymbol{P}, x) \text { for } i=1 \ldots 5
$$


where $G_{i}$ denotes the newly introduced performance function, and $x_{\min }$ and $x_{\max }$ denote the lower and upper bounds of the x-coordinate, respectively. System reliability is used here and five performance functions are defined in Equation (7). Therefore, the procedure described in Equation (8) is applied five times.

\section{SOLUTION STRATEGY}

Defect probability is expressed as a probability of failure, and a reliability algorithm is thus used. The proposed procedure needs to deal efficiently with the two following points.

- An optimization problem must be solved each time a performance function is evaluated, as indicated in Equation (8). A design of experiments is performed with respect to the parameters of the boundary conditions, i.e. the contact force and the coefficient of friction. Response surfaces are calibrated and subsequently used to identify the minimum of the performance function with a reduced number of calls to the finite element model. The procedure is described in Section 4.

- The total number of calls to the performance functions should be reduced since a non-linear FE model is involved. An advanced procedure, based on AK methods, is implemented to determine the defect probability with reduced numerical efforts; this algorithm is described in details in Section 4

\section{Evaluation of the performance functions}

The identification of the minimum of the performance function expressed by Equation (8) is numerically demanding. A non-linear finite element simulation is required, and the identification of the minimum is thus numerically prohibitive.

Surrogate models are used to reduce the computational efforts associated with Equation (8). A response surface is calibrated for each performance function and 
for each realization of the random variables (generated by the reliability algorithm). The response surfaces involve as input parameters the coefficient of friction and the force applied as a boundary conditions. The calibration set consists of nine samples selected such that they cover the range of variation of these parameters. In the case where the force $F$ is near its lower bound, the coefficient may vary over a large range of values, and hence the design of experiments points are scattered. In the case where the applied force is near its upper bound, the coefficient of friction varies over a narrower range, and the design of experiments points are concentrated. Finite element simulations are performed using realizations of the random variables generated by the reliability algorithm, which define the geometry of the model. For each realization, nine simulations are performed; the boundary conditions are set for the nine pairs $\left(\mu^{(k)}-F^{(k)}\right), k=1 \ldots 9$, as shown in Figure 10. The position in the wiper is not considered at this stage and only the parameters associated with the boundary conditions (i.e. $\mu$ and $F)$ are involved in this design of experiments. In case the reliability procedure is performed using $N$ samples; $9 N$ finite element simulations are performed in total.

For each realization of the random variables, the performance functions $g_{i}, i=$ $1 \ldots .5$ are subsequently approximated by second order polynomials:

$$
g_{i}\left(\boldsymbol{D}^{(j)}, \boldsymbol{P}^{(j)}, x\right) \simeq Q_{i}^{(j)}(F(x), \mu(x))
$$

where $Q_{i}^{(j)}$ denotes second order polynomials. Unlike surrogate model based reliability algorithms (see e.g. (Bucher and Bourgund 1990)), the polynomials $Q_{i}^{(j)}$ are not expressed with respect to the random variables and the polynomial coefficient need to be determined for each realization. The experience showed that the responses of the finite element model are quasi-linear with respect to the 
force and the coefficient of friction (for the considered range of variation of these parameters). Therefore, second order polynomials provide a suitable framework to approximate the mechanical response, as they account for the linear trend and the slight non-linearities. These polynomials are solely used to reduce the efforts associated with the optimization problem described in Equation (8).

The quality of the polynomial fit is checked using cross-validation. The applied strategy consists of excluding a sample-result pair from the calibration set, then calibrating the response surface using the remaining data and using it to predict the outcome of the finite element model associated with the excluded sample. As the response is known, the error associated with the surrogate model can be estimated. The method is repeated multiple times, for all the nine design of experiments samples and for multiple realizations of the random variables. The coefficient of determination for prediction $R^{2}$ is determined; it provides an appropriate metric of the quality of the fit (Myers et al. 2008). All the design of experiments points shown in Figure 10 are tested for 100 independent realizations of the random variables, and we obtain $R^{2}>0.99$ for all the performance functions. It can be concluded from the high $R^{2}$ values that second-order polynomials accurately approximate the response of the finite element models, and in the following all nine design of experiments samples are used for the calibration of the response surface.

\section{Reliability analysis using AK methods}

Probability evaluation using sampling techniques consists in classifying a large population (obtained using Monte Carlo sampling or any equivalent procedure) into safe and unsafe realizations according to the sign of the performance function $g(\mathbf{x})$, where $\mathbf{x}$ denotes the random variables, i.e. $\mathbf{x}^{T}=\left[\boldsymbol{D}^{T}, \boldsymbol{P}^{T}\right]$. A schematic 
representation of the classification between the failure domain and the safe domain is shown in Figure 11a. Such approaches can become prohibitively expensive in terms of computational effort, especially for low probability evaluations and/or complex numerical performance functions. A possible solution consists of replacing the latter with a surrogate model that can be evaluated inexpensively. Active learning and Kriging based methods (AK) use Kriging in an iterative procedure to build a separator of safe and unsafe realizations with only a few well-chosen performance function evaluations. The AK-SYS method (Fauriat and Gayton 2014 ) is used in this application, since a system reliability problem is involved. This algorithm is an adaptation of the AK classification principle for system reliability evaluation based on Monte Carlo simulation. The classification of the AK-method is decomposed into 5 steps (Echard et al. 2011):

1. Generation of a Monte Carlo population $S$ of size $n_{M C}: \mathbf{x}^{(1)} \ldots \mathbf{x}^{\left(n_{M C}\right)}$. At this stage none of them are evaluated on the mechanical performance function.

2. Definition of the initial design of experiments by randomly select $N$ points in $S$. Compute all of the $N$ points on the mechanical model.

3. Computation of the Kriging surrogate model according to the design of experiments;

4. Prediction by Kriging on $S$ and estimation of the probability of failure;

5. Identification of the best next point in $S$ to evaluate on the performance function if the stopping criterion is not yet reached.

A schematic representation of the AK method is shown in Figure 11b.

The main originality of the AK method is the preliminary choice of the population $S$. In the case of a unique performance function, the learning criterion is 
defined by:

$$
U\left(\mathbf{x}^{(j)}\right)=\frac{\left|\hat{g}\left(\mathbf{x}^{(j)}\right)\right|}{\sigma_{g}\left(\mathbf{x}^{(j)}\right)} \text { for } j=1: n_{M C}
$$

where $\hat{g}\left(\mathbf{x}^{(j)}\right)$ and $\sigma_{g}\left(\mathbf{x}^{(j)}\right)$ are respectively the Kriging prediction and standard deviation estimation. Under Gaussian assumptions, $U\left(\mathbf{x}^{(j)}\right)$ is linked to the probability that $\hat{g}\left(\mathbf{x}^{(j)}\right)$ would not have the same sign as $g\left(\mathbf{x}^{(j)}\right)$. The stopping criterion is reached when all the points of $S$ are considered to be well classified, i.e. when $\min \left(\mathbf{x}^{(j)}\right)>2$. For system reliability, the AK-SYS method (Fauriat and Gayton 2014) deals with $m$ different performance functions $g_{j}(\mathbf{x})$ and union probability is required:

$$
P_{f}=\operatorname{Prob}\left(g_{1}(\mathbf{x}) \leq 0 \bigcup \ldots \bigcup g_{m}(\mathbf{x}) \leq 0\right)
$$

The AK-SYS method is based on the AK-classification principle and the follow enrichment criterion is adopted as discussed in (Fauriat and Gayton 2014):

$$
U\left(\mathbf{x}^{(j)}\right)=\frac{\left|\hat{g}_{s}\left(\mathbf{x}^{(j)}\right)\right|}{\sigma_{g_{s}}\left(\mathbf{x}^{(j)}\right)} \text { for } j=1: n_{M C}
$$

where $s$ is the performance function index that minimizes $g_{j}(\mathbf{x})$. The advantage procured by this approach is that no calls (or only a small number) will be made to the true performance functions that have little or no influence on the system failure domain.

In the implementation of the procedure, a constant trend is used for the Kriging surrogate model, together with a Gaussian isotropic covariance function. Therefore, three hyperparameters are used in total: the mean value, the variance and the correlation length. They are identified using the method of the maximum of likelihood.

Figure 12 represents the workflow implemented for the reliability analysis; it can be described as a double loop approach. The outermost loop is the AK- 
based reliability method, which iteratively generates realizations of the random variables. The innermost loop evaluates the performance functions associated with the samples generated by the AK method. These functions also involve the $\mathrm{x}$-coordinate. For each realization, a response surface is calibrated and used to eliminate this additional variable.

\section{RESULTS}

Figure 13 shows the variation in defect probability as the AK method is used. In total, approx. 1300 finite element simulations are required to run the procedure, as multiple analyses are performed for each evaluation of the performance functions, as discussed in Section 4.

During the first few iterations of the procedure, the design of experiments does not include enough samples and the Kriging surrogate models have low fidelity. As a result, the confidence interval associated with the defect probability is wide. During the subsequent iterations, the design of experiments is enriched with additional samples, which improves the fidelity of the surrogate models and reduces the width of the confidence intervals.

Defect probability remains below the predefined quality threshold, indicated by a dashed line in Figure 13. The procedure is stopped prematurely, even though it has not fully converged. Indeed, multiple nonlinear analyses are required, which causes excessive numerical efforts. The defect probability is smaller than its threshold value and the confidence interval does not include this threshold value either. It can hence be concluded that the quality requirements are met and no additional simulation is performed. The failure probability is determined by counting the total number of samples in the failure domain using the surrogate model (i.e. the samples with $\hat{g}(\mathbf{x}) \leqslant 0$ ). It is assumed that the sample $\mathbf{x}^{(j)}$ is very likely to be well classified in case $U\left(\mathbf{x}^{(j)}\right) \geqslant 2$, the relevance of this threshold 
value is discussed e.g. in (Echard et al. 2011). This criterion is used to determine the confidence bounds of the failure probability. The lower bound of the failure probability is obtained using the samples in the failure domain with a low chance of misclassification, i.e. the samples with $\hat{g}(\mathbf{x}) \leqslant 0$ and $U(\mathbf{x}) \geqslant 2$; the upper bound of the failure probability is determined using the samples in the safe domain with a low chance of misclassification, i.e. the samples with $\hat{g}(\mathbf{x})>0$ and $U(\mathbf{x}) \geqslant$ 2 .

The method also enables us to identify the dominant failure mode. This information may be used as an input for quality improvement.

\section{CONCLUSIONS}

A procedure for the tolerance analysis of a deformable system is proposed in this study. The method is used to determine the defect probability associated with an industrial problem: a wiper blade manufactured by Valeo Wiper Systems. The distributions of the uncertain dimensions are estimated directly from measurements obtained from the factory. The performance of the system is obtained from the structural response. A finite element model is of the rubber blade is prepared; it includes uncertainties in the geometry of the profile. Structural reliability methods are subsequently used to compute the defect probability. The problem is formulated using a double loop approach: the outermost loop consists of the reliability analysis and realizations of the random variables are generated. The innermost loop consists of solving an optimization problem for each realization of the random variables. The numerical efforts are reduced using a surrogate model based procedure. In the innermost loop, design of experiments is used for each sample and the structural responses are approximated by second order polynomials. The so-called AK methods are used to reduce the numerical efforts associated with the reliability analysis; such algorithm rely on 
the use of Kriging surrogate models. It is concluded from this study that quality requirements are met by the wiper blade.

Two major original features are introduced in this study. Firstly, the distributions associated with the dimensions are directly identified from their realizations, obtained from parts collected on the production lines. This strategy also provides the correlation between the dimensions, which is important information that designers often lack. It is necessary to adopt such an approach, based on industrial data, to obtain a realistic estimation of the defect probability. Secondly, the approach is directly deployed on a full scale industrial model. Because of the considerable uncertainties in the dimensions, the stiffness matrix cannot be assumed to be constant, and the influence method is not applicable. A structural reliability algorithm is used instead, and defect probability is computed using AK methods.

Future work is geared towards improvements of the geometrical modeling of the wiper blade by considering form defects.

\section{ACKNOWLEDGEMENTS}

This research is partially supported by the French Science Agency (ANR) under contract No. ANR-11-MONU-013, which is gratefully acknowledged by the authors.

The authors are grateful to Laurent André-Masse and Sébastien Jallet, Valeo VWS, for their collaboration on this study and their assistance with the finite element model. David Turner is gratefully acknowledged for the proofreading of the manuscript.

\section{REFERENCES}

Akaike, H. (1974). "A new look at the statistical model identification." IEEE Transactions on Automatic Control, 19(6), 716-723. 
Bucher, C. and Bourgund, U. (1990). "A fast and efficient response surface approach for structural reliability problems." Journal of Structural Safety, 7(1), 57-66.

Chase, K. W. and Parkinson, A. R. (1991). "A survey of research in the application of tolerance analysis to the design of mechanical assemblies." Research in Engineering Design, 3(1), 23-37.

Dahlström, S. and Lindkvist, L. (2006). "Variation simulation of sheet metal assemblies using the method of influence coefficients with contact modeling." Journal of Manufacturing Science and Engineering, 129(3), 615-622.

Dantan, J.-Y. and Qureshi, A.-J. (2009). "Worst-case and statistical tolerance analysis based on quantified constraint satisfaction problems and Monte Carlo simulation." Computer-Aided Design, 41(1), 1 - 12.

Dumas, A., Gayton, N., Dantan, J.-Y., and Sudret, B. (2015). "A new system formulation for the tolerance analysis of overconstrained mechanisms." Probabilistic Engineering Mechanics, 40, 66 - 74 .

Echard, B., Gayton, N., and Lemaire, M. (2011). "AK-MCS: an active learning reliability method combining kriging and monte carlo simulation." Structural Safety, 33, 145-154.

Echard, B., Gayton, N., Lemaire, M., and Relun, N. (2013). "A combined importance sampling and kriging reliability method for small failure probabilities with time-demanding numerical models." Reliability Engineering \& System Safety, 111, $232-240$.

Evans, D. (1975). "Statistical tolerancing: The state of the art. part ii, methods for estimating moments." Journal of Quality Technology, 17(1), 1-12.

Fauriat, W. and Gayton, N. (2014). "AK-SYS: An adaptation of the AK-MCS method for system reliability." Reliability Engineering \& System Safety, 123(0), $137-144$. 
Gayton, N., Beaucaire, P., Bourinet, J.-M., Duc, E., Lemaire, M., and Gauvrit, L. (2011). "APTA: Advanced probability - based tolerance analysis of products." Mécanique et Industrie, 12, 71-85.

Gordis, J. and Flannelly, W. (1994). "Analysis of stress due to fastener tolerance in assembled components." AIAA Journal, 32, 2440-2446.

Greenwood, W. and Chase, K. (1987). "A new tolerance analysis method for designers and manufacturers." Transaction of the ASME, 109-116.

Hong, Y. and Chang, T. (2002). "A comprehensive review of tolerancing research." International Journal of Production Research, 40(11), 2425-2459.

Karhunen, K. (1947). "Über lineare Methoden in der Wahrscheinlichkeitsrechnung." Amer. Acad. Sci., Fennicade, Ser. A, 37, 3-79.

Lemaire, M. (2010). Structural Reliability. ISTE, Wiley.

Li, Z., Yue, J., Kokkolaras, M., Camelio, J. A., Papalambros, P., and Hu, J. S. (2004). "Product tolerance allocation in compliant multistation assembly through variation propagation and analytical target cascading." 2004 ASME International Mechanical Engineering Congress and Exposition, Anaheim, CA (November 1320).

Lindau, B., Lorin, S., Lindkvist, L., and Söderberg, R. (2015). "Efficient contact modeling in nonrigid variation simulation." Journal of Computing and Information Science in Engineering, 16(1), 011002.

Liu, P.-L. and Der Kiureghian, A. (1986). "Multivariate distribution models with prescribed marginals and covariances." Probabilistic Engineering Mechanics, $1(2), 105-112$.

Liu, S. and Hu, S. (1996). "Variation simulation for deformable sheet metal assemblies using finite element methods." Journal of Manufacturing Science and Engineering, 119(3), 368-374.

Liu, S. C. and Hu, S. J. (1997). "Variation simulation for deformable sheet metal 
assemblies using finite element methods." ASME Journal of Manufacturing Science and Engineering, 119(3), 368374.

Loève, M. (1977). Probability Theory. Springer-Verlag, New York, $4^{\text {th }}$ edition edition.

Mai, J.-F. and Scherer, M. (2012). Simulating Copulas (Stochastic Models, $\underline{\text { Sampling Algorithms and Applications), Vol. } 4 \text { of Series in Quantitative }}$ Finance. World Scientific.

Myers, R. H., Montgomery, D. C., and Andersson-Cook, C. M. (2008). Response surface methodology: process and product optimization using designed experiments. Wiley, Jersey, USA.

Nigam, S. and Turner, J. (1995). "Review of statistical approaches to tolerance analysis." Computer-Aided Design, 27(1), 6 - 15.

Qureshi, A.-J., Dantan, J.-Y., Sabri, V., Beaucaire, P., and Gayton, N. (2012). "A statistical tolerance analysis approach for over-constrained mechanism based on optimization and monte carlo simulation." Computer Aided Design, 44(2), $132-142$.

Roy, U., Liu, C., and Woo, T. (1991). "Review of dimensioning and tolerancing: representation and processing." Computer-Aided Design, 23(7), 466 - 483.

Scholtz, F. (1995). "Tolerance stack analysis methods." Report no., Boeing Technical Report.

Schölzel, C. and Friederichs, P. (2008). "Multivariate non-normally distributed random variables in climate research \&ndash; introduction to the copula approach." Nonlinear Processes in Geophysics, 15(5), 761-772.

Söderberg, R., Lindkvist, L., and Dahlström, S. (2006). "Computer-aided robustness analysis for compliant assemblies." Journal of Engineering Design, 17(5), $411-428$. 
672 LIST OF FIGURES 


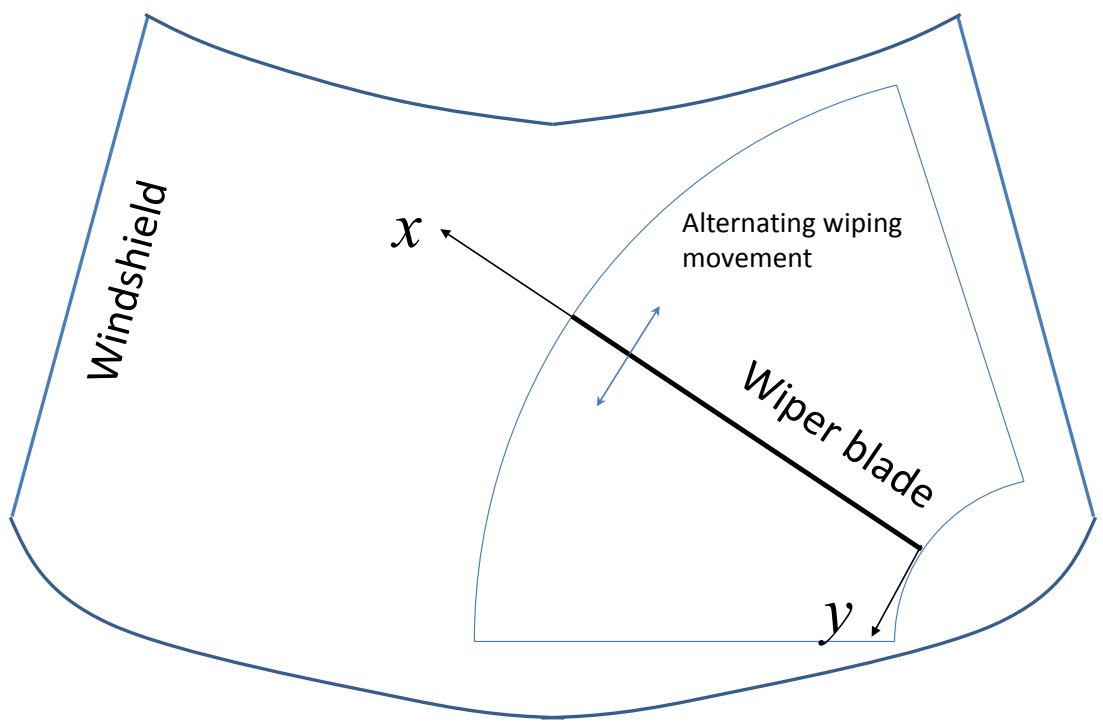

FIG. 1: Alternating movement of the wiper system. The local coordinate system, associated with the wiper blade, is indicated by the arrows. 


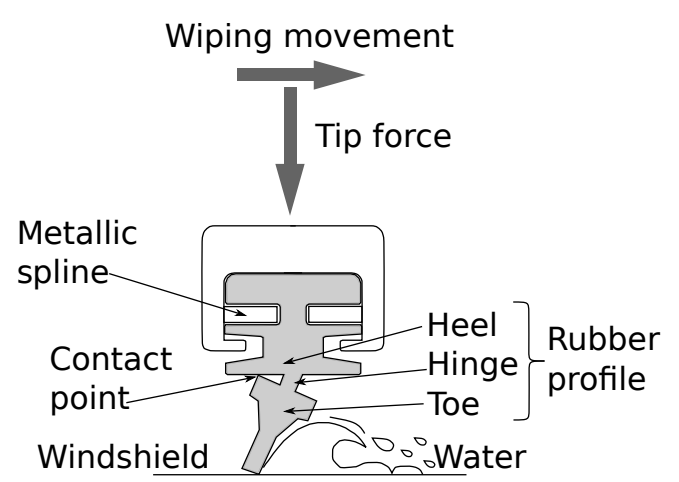

(a)

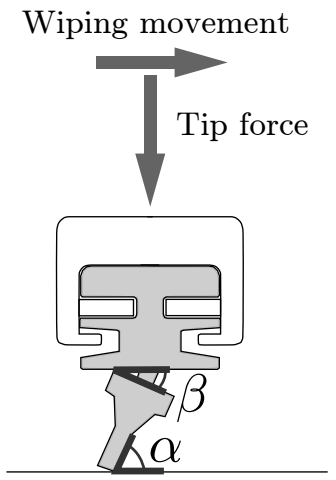

(b)

FIG. 2: Blade profile. (a) Description of the profile. (b) Functional requirements associated with the profile. 


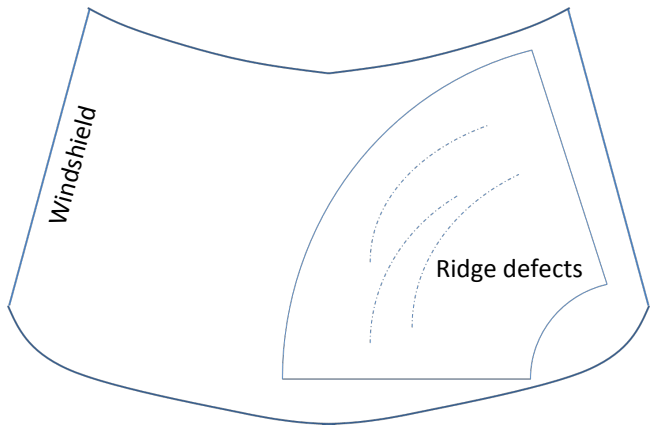

(a)

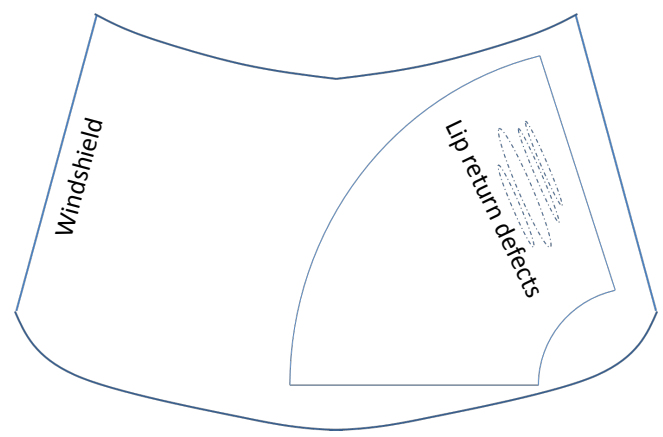

(b)

FIG. 3: Wiping defects 


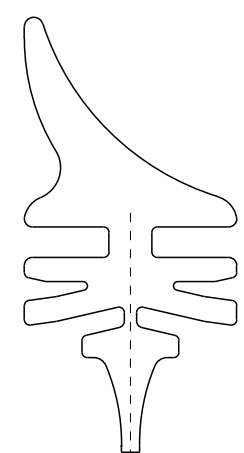

(a)

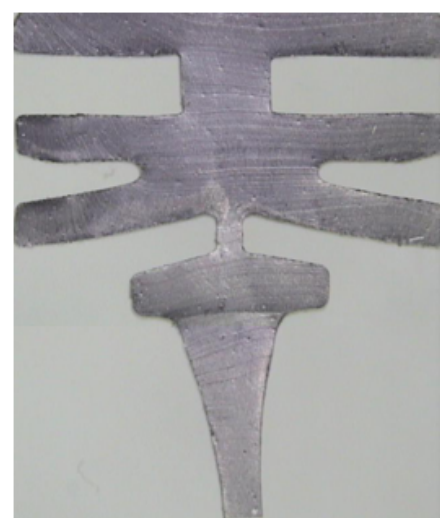

(b)

FIG. 4: (a) Reference cross-section of the blade as it appears in the drawings of the component. (b) Cross-section of a blade as obtained after extrusion (image courtesy of Valeo, copyright Valeo). 


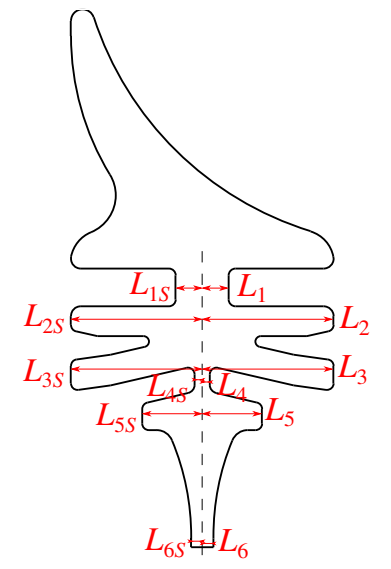

(a)

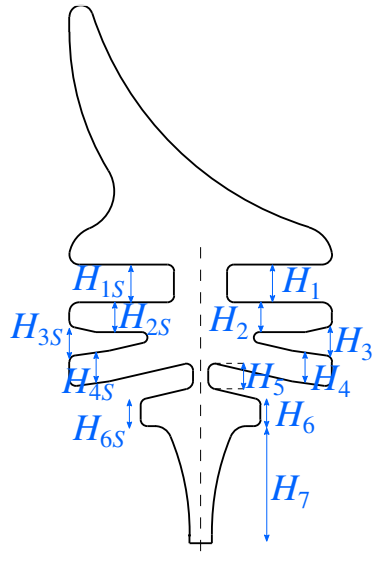

(b)

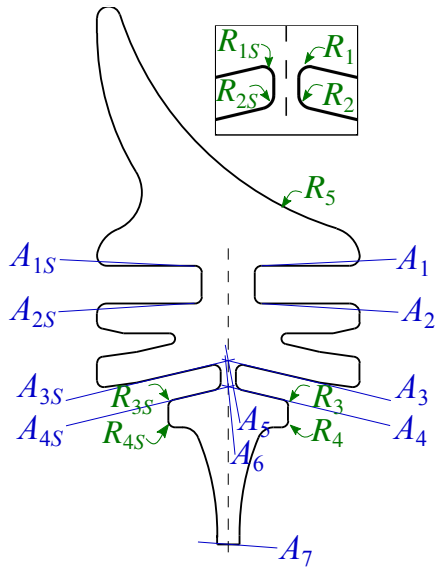

(c)

FIG. 5: Parameterization of the reference cross-section to take into account manufacturing uncertainties.(a) Length of subcomponents. (b) Height of subcomponents. (c) Angles and fillet radii. 


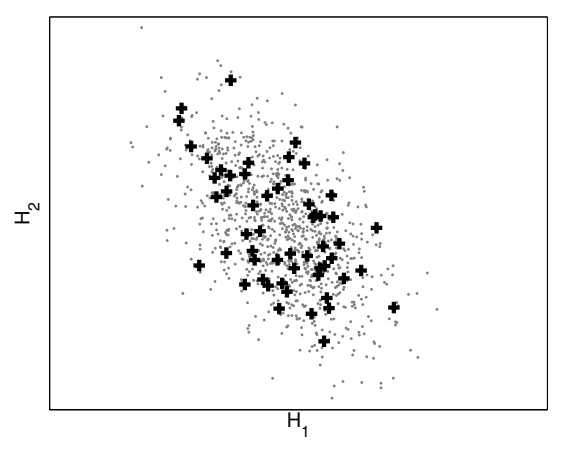

(a)

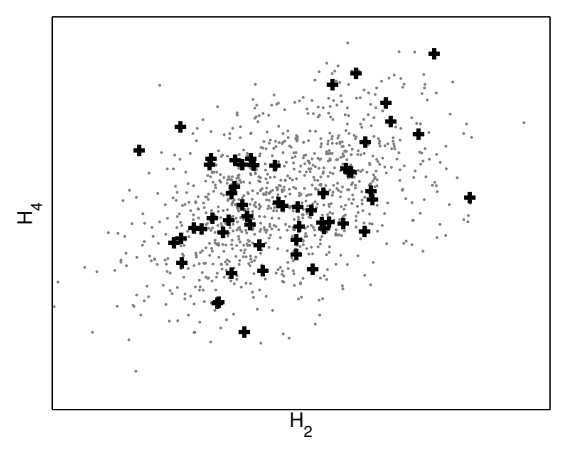

(b)

FIG. 6: Correlation between the dimensions. The black crosses represent the data measured on parts and the grey points represent 1000 samples generated using Monte Carlo simulation. (a) $H_{1}$ and $H_{2}$. (b) $H_{2}$ and $H_{4}$. 


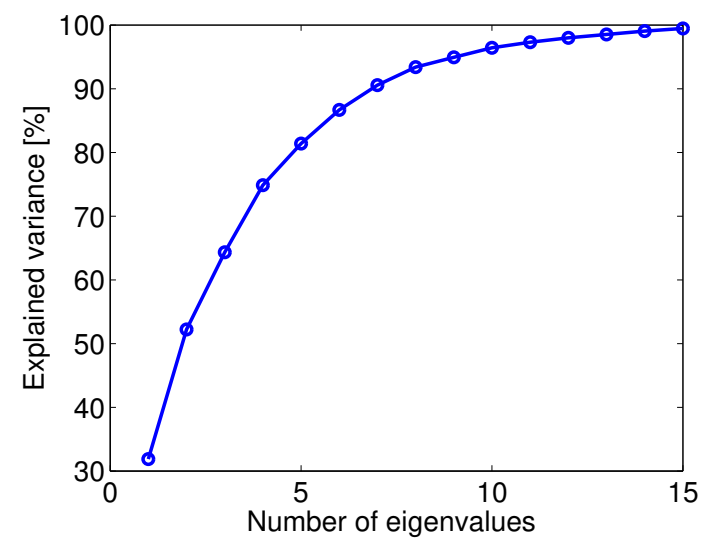

FIG. 7: Explained variance in terms of the number of eigenvalues used in the Karhunen-Loève expansion. 


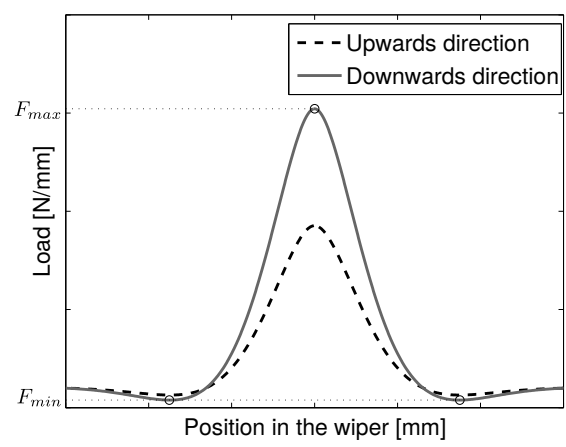

(a)

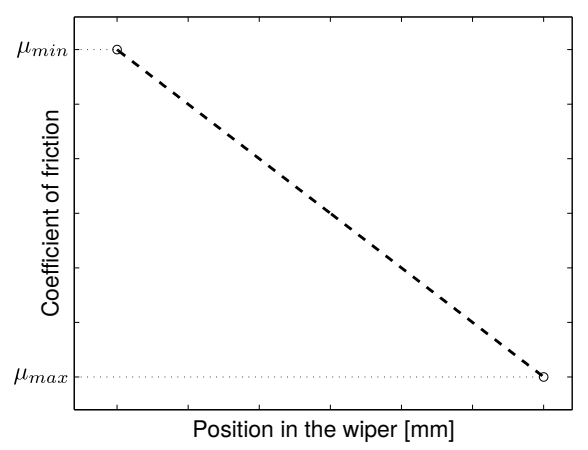

(b)

FIG. 8: (a) Variation in force with respect to position along the wiper blade.

(b) Variation in the coefficient of friction between the blade and the windshield, expressed with respect to the position along the wiper blade. 


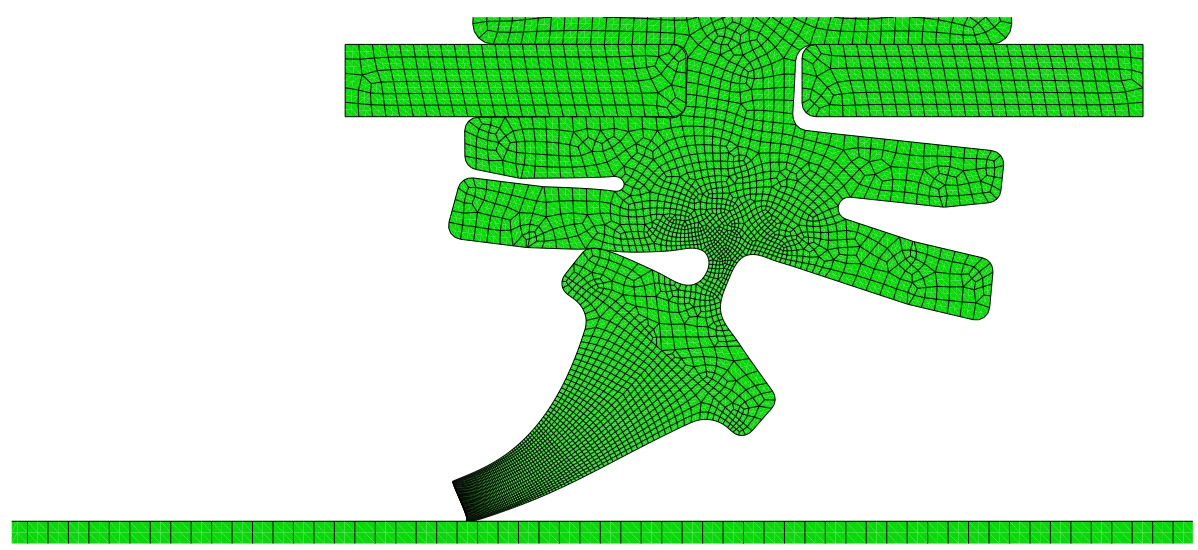

FIG. 9: Finite element model of the blade profile. 


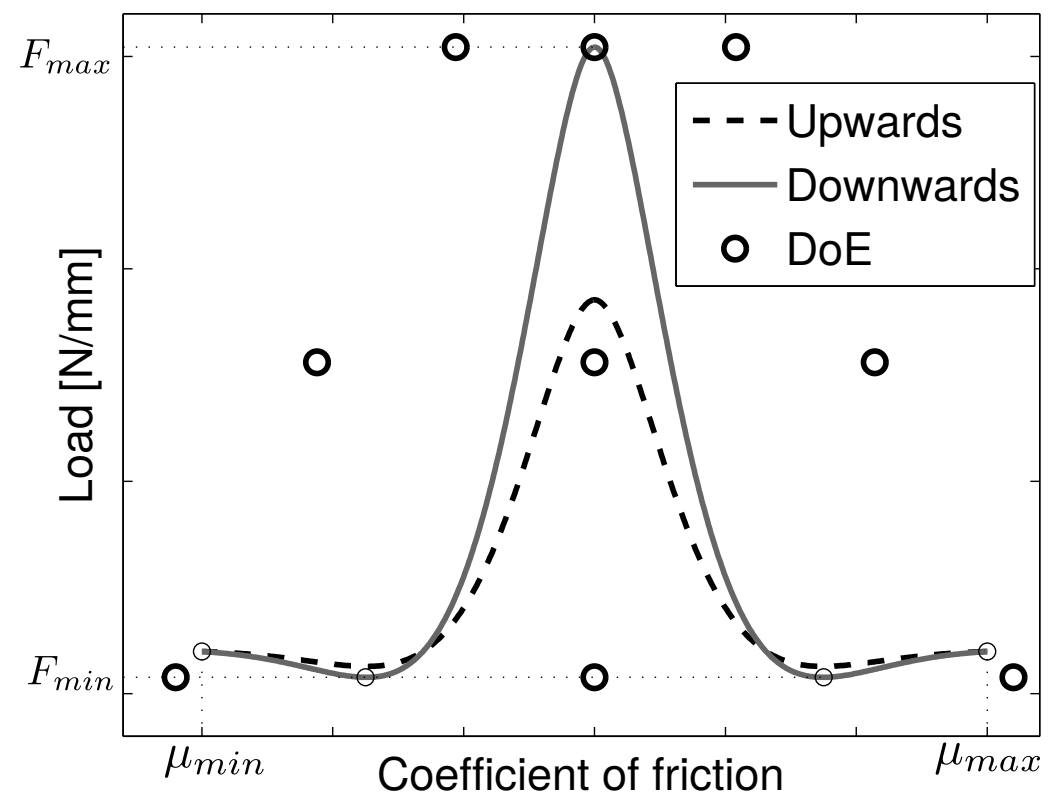

FIG. 10: Generation of the calibration set used for the response surfaces (hollow points). 


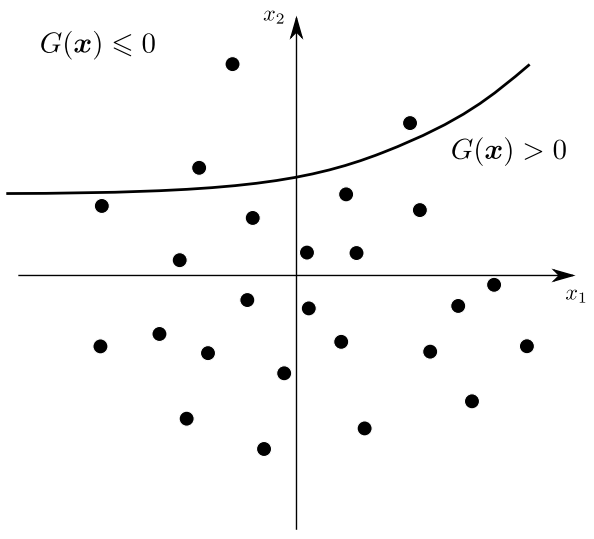

(a)

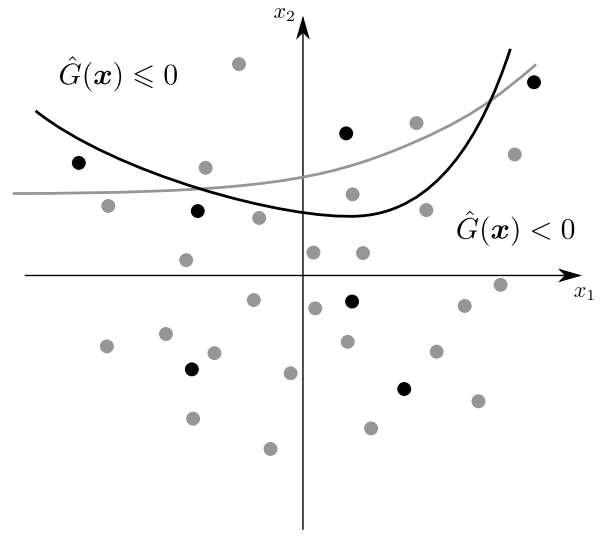

(b)

FIG. 11: Schematic representation of the reliability procedures. (i) Classification of samples (represented by the black points) into failure domain and safe domain. The thick black line represents the limit state. (b) Basic concept of the AK methods. The black points represent the enriched points where the performance function is evaluated; the gray points represent the samples where the surrogate model is used instead. The gray line represents the actual limit state, the black line represents the approximate limit state obtained using the surrogate model and used for the classification. 


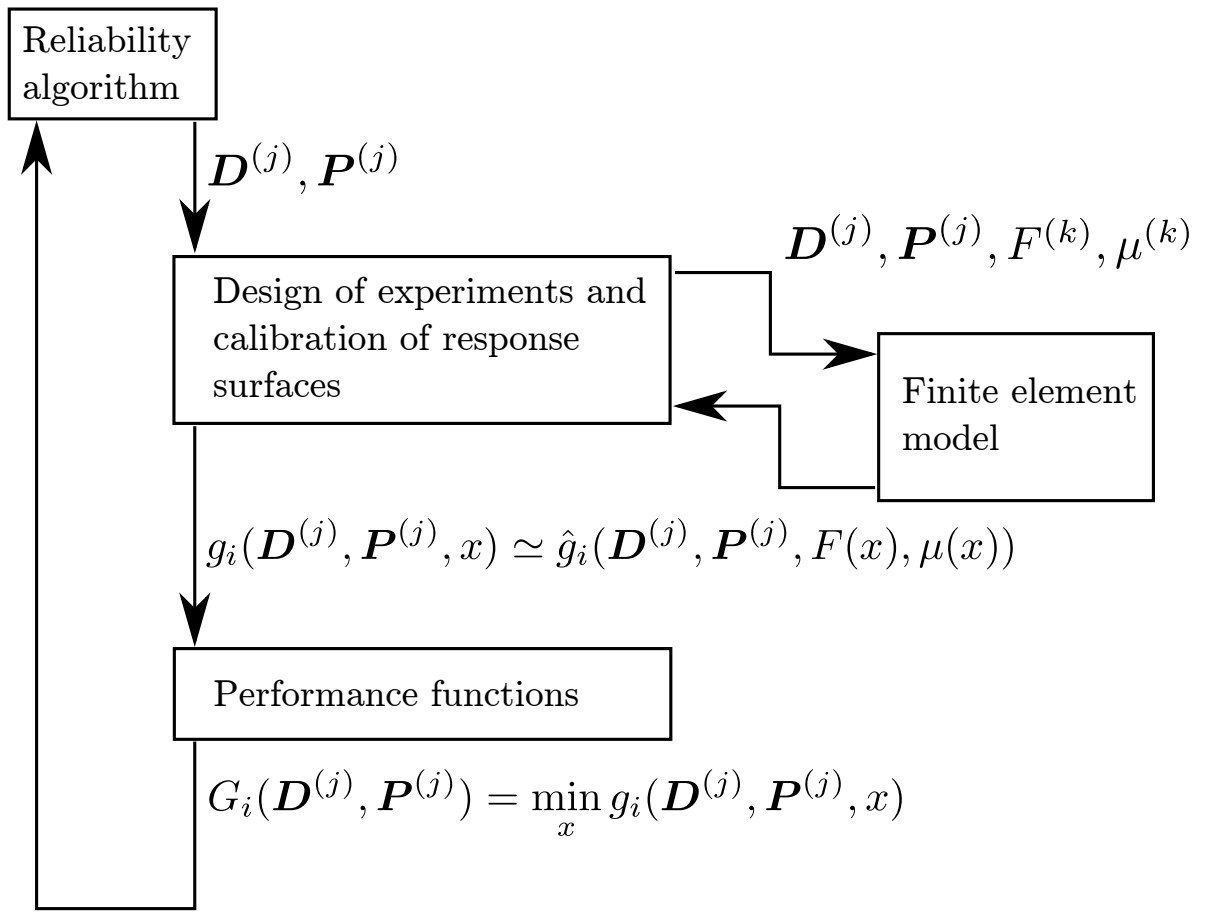

FIG. 12: Workflow of the procedure. 


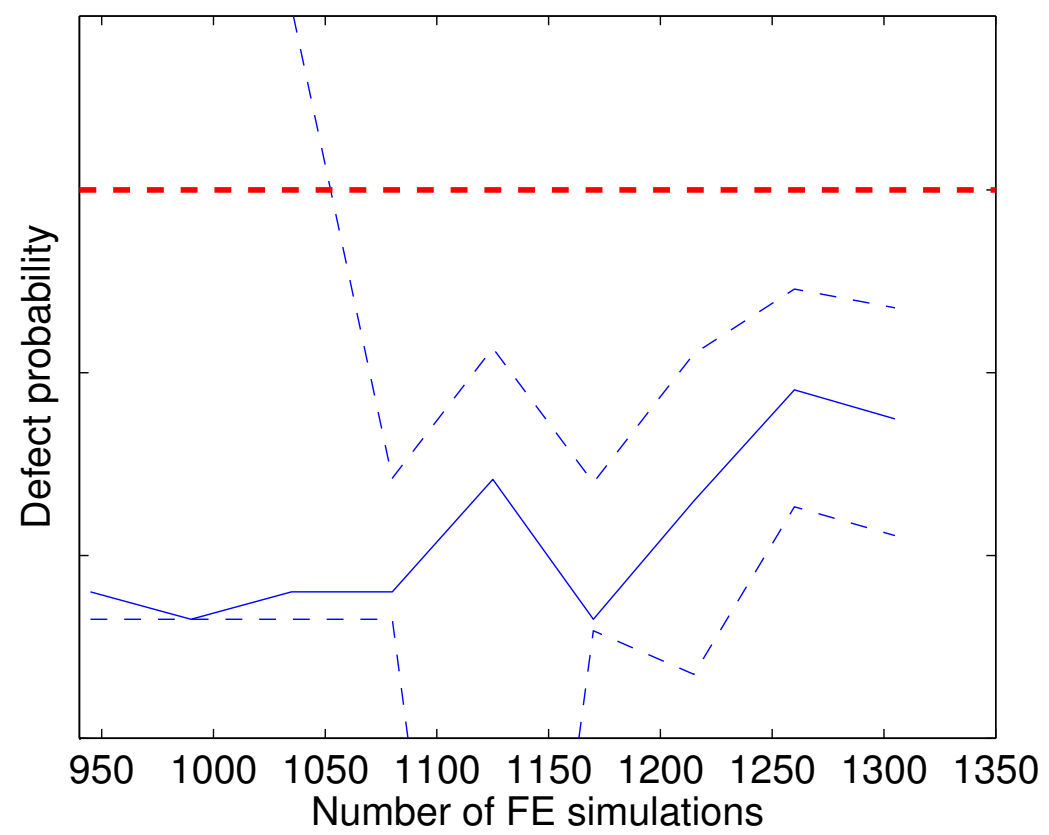

FIG. 13: Variation in defect probability (solid line) during the iterations of the AK method. The dashed line represents the confidence bounds of the defect probability; the thick dashed line represents the upper bound of the defect probability defined by the quality standards. 